\title{
Crucial role of orbital structure in formation of frustrated magnetic structure in $\mathrm{BiMnO}_{3}$
}

\author{
L. E. Gonchar ${ }^{1,2, *}$ and A. E. Nikiforov ${ }^{2}$ \\ ${ }^{1}$ Ural State University of Railway Transport, 66 Kolmogorov St., Ekaterinburg 620034, Russia \\ ${ }^{2}$ Ural Federal University, 19 Mira St., Ekaterinburg 620002, Russia
}

(Received 6 March 2013; revised manuscript received 18 June 2013; published 3 September 2013)

\begin{abstract}
The paper presents an investigation in the field of orbital physics of strongly correlated oxides. The theoretical study of vibronic mechanism of orbital and magnetic structures forming in $\mathrm{BiMnO}_{3}$ crystal is carried out. An effect of orbital structure upon superexchange interaction is described. Nonlinear and second-neighbor terms in vibronic interaction on manganese ions play an important role in magnetic ordering of frustrated $\mathrm{BiMnO}_{3}$. It is shown that the linear vibronic interaction is insufficient to describe the experimentally detected ferromagnetic structure of bismuth manganite. The new approach to orbital structure formation, presented in the paper, could be used not only in manganite physics but also in other Jahn-Teller compounds.
\end{abstract}

DOI: 10.1103/PhysRevB.88.094401

PACS number(s): 75.47.Lx, 75.85.+t, 75.25.Dk, 75.30.Et

\section{INTRODUCTION}

$\mathrm{BiMnO}_{3}$ crystal is a vivid example of renewal of interest in manganite crystals. The manganite crystals $R \mathrm{MnO}_{3}(R=\mathrm{La}$, $\mathrm{Nd}, \mathrm{Pr}, \mathrm{Dy}, \mathrm{Sm}, \mathrm{Tb}, \mathrm{Gd}, \mathrm{Ho}, \mathrm{Y}$ ) are parent compounds to colossal magnetoresistance crystals and have a peculiar interdependence between charge, lattice, orbital, and magnetic degrees of freedom. A lot of investigations were devoted to the multiferroic (MF) manganites, ${ }^{1}$ a combination of magnetic ordering with ferroelectricity (FE). The compound without orbital degeneracy $\mathrm{BiFeO}_{3}$ is also a good MF crystal. ${ }^{2}$ The mechanisms of $\mathrm{FE}$ in these crystals $\left(R \mathrm{MnO}_{3}\right.$ and $\left.\mathrm{BiFeO}_{3}\right)$ are different: ${ }^{3,4}$ in magnanites, there is a spiral spin structure on manganese sublattice and in the bismuth oxide, a lone pair of electrons of $\mathrm{Bi}^{3+}$ ion creates the dipole moment. The MF behavior of ferromagnetic $\mathrm{BiMnO}_{3}$ was discovered in films ${ }^{5}$ and polycrystals, ${ }^{6}$ but in a single crystal, the FE is impossible due to symmetry considerations., ${ }^{4,7}$ The crystal structure of $\mathrm{BiMnO}_{3}$ with $C 2$ (No. 5) space symmetry is determined in earlier investigations of Refs. 8 and 9. This symmetry group is noncentrosymmetric and allows the coexistence of ferromagnetism (FM) and FE. In Refs. 10 and 11, the later experimental studies of this compound disclaim the noncentrosymmetric space group of this crystal structure and describe the structure by the centrosymmetric space group $C 2 / c$ (No. 15). This symmetry allows no coexistence of FE and FM in the crystal. The detailed experimental comparison of both symmetries was made in Ref. 12. The conclusion of $C 2 / c$ symmetry was made by the authors. The FM magnetic ordering is reliably determined by experiments, ${ }^{10,11,13,14}$ thus the FE of the single crystal is not ascertained. The Curie temperature of $\mathrm{BiMnO}_{3}$ is $T_{C} \sim 100 \mathrm{~K} .^{6,8-13}$ The temperature of possible $\mathrm{FE}$ phase transition is $T_{\mathrm{FE}}=450 \mathrm{~K}$ (Refs. 6 and 15 ) or $770 \mathrm{~K}$ (Refs. 13 and 15). The last value is a result of indirect measurements and is rejected in later investigations. ${ }^{12}$ Theoretical and experimental investigations of orbital and magnetic orderings are carried out in order to find if it is possible to switch this crystal into the MF state.

The orbital ordering (OO) of $\mathrm{BiMnO}_{3}$, estimated by lattice distortion, is not the same type with the $\mathrm{OO}$ in $\mathrm{LaMnO}_{3}$ manganites. ${ }^{7,8,11-15}$ It is formed by interaction of an orbitally degenerated manganese ions sublattice and a strongly polarized bismuth ions sublattice. ${ }^{16}$ The temperature of $\mathrm{OO}$ is proposed to be $T_{\mathrm{OO}}=750 \mathrm{~K}$ (Refs. 10 and 15) or $450 \mathrm{~K}$ (Refs. 13 and 15). The orbital structure of oxygen octahedra is complicated due to Jahn-Teller distortion. Within each pseudoperovskite direction, the $e_{g}$ orbital sequence is $d_{3 x^{2}-r^{2}} / d_{3 y^{2}-r^{2}} / d_{3 x^{2}-r^{2}} / d_{3 z^{2}-r^{2} \ldots . .}{ }^{8-12,15}$ The orbital structure changes with the crystal structure, which undergoes some phase transition with temperature and external pressure, ${ }^{6-14,17}$ thus, the Jahn-Teller distortions of oxygen octahedra are suppressed by growing temperature and pressure. The theoretical calculation of the orbital structure was carried out in Ref. 18 with the same result: the directions of $d$ orbitals coincide with the elongated $\mathrm{Mn}-\mathrm{O}$ bonds. Thus the theoretical studies of $\mathrm{BiMnO}_{3}$ orbital structure were carried out only as analysis of experimentally determined Jahn-Teller distortions of nearest-neighbor oxygen coordination.

The description of the magnetic structure due to $\mathrm{OO}$ is based generally on Goodenough-Kanamory rules. ${ }^{8-12}$ The $a b$ initio calculations of exchange interaction were made by Soloviev et al. in Refs. 7, 16, and 18. In order to find the possibility of FE, the authors propose a model of a complicated canted magnetic structure. Nevertheless, this ordering is inconsistent with experiments confirming collinear FM structure. Multiple magnetic transitions are found under external pressure. ${ }^{11,14,19}$ There is no detailed theoretical analysis of the mechanism of ferromagnetic to antiferromagnetic (AFM) transition of $\mathrm{BiMnO}_{3}$ under pressure.

Like rare-earth manganites, $\mathrm{BiMnO}_{3}$ changes crystal, orbital, and magnetic properties with doping. The nonstoichiometry by oxygen ${ }^{20}$ leads to crystal symmetry changes, including $C 2$ space group, FM and AFM structures. There is an ongoing discussion of FE possibility in the nonstoichiometric samples. Other kinds of doping used by the authors are rare-earth and alkaline-earth dopings like in manganites. ${ }^{21}$ Similar to lanthanum-manganite-based compounds, the charge ordered states, metallic behavior, and AFM structures are detected in these investigations.

The effect of additional interactions in the vibronic Hamiltonian upon orbital structures was proposed in Ref. 22 for layered manganites and in Ref. 23 for titanates. These investigations demonstrate the significance of a thorough description of orbital structure and the necessity to advance 
the orbital structure model presented earlier for manganites in Refs. 24 and 25.

The aim of this paper is to present an advanced model of orbital structure in order to describe a vibronic mechanism of magnetic structure forming in the case of magnetic frustration. Taking into account the linear vibronic interaction of orbitally degenerated manganese subsystem not only with nearest-neighbor oxygen coordination, but with next-nearest neighboring bismuth environment and additional nonlinear vibronic terms corresponding to nearest neighbors, the FM ordering of bismuth manganite is described. The FM state is a result of orbital degeneration of manganese sublattice and thus is impossible for $\mathrm{BiFeO}_{3}$, which is a $G$-type AFM crystal. $^{2}$ The FM ordering combined with an FE state promises a wide range of technical applications of the crystal. In spite of the prohibition of this combination of properties for bulk $\mathrm{BiMnO}_{3}$ crystal, the investigation of the bulk crystal plays an important role in the description of thin-film orbital and magnetic structures.

\section{VIBRONIC INTERACTIONS AND ORBITAL STRUCTURE}

The crystal structure of $\mathrm{BiMnO}_{3}$ is determined in the experimental studies of Refs. 10 and 11, and it belongs to the $C 2 / c$ space symmetry group. The lattice constants and crystal structure angles are, correspondingly, $a=9.5473 \AA$, $b=5.6167 \AA, c=9.8699 \AA, \alpha=\gamma=90^{\circ}$, and $\beta=110.66^{\circ}$. The crystal structure parameters are shown in Table I.

The monoclinic coordinate system in the crystal is of nonCarthesian type. That is why a pseudoperovskite reference frame is used throughout the paper. The correlation between coordinate axes of different referent frames is described in detail in Appendix.

The crystal structure is of distorted perovskite type. In $\mathrm{BiMnO}_{3}$ crystal, $\mathrm{Mn}^{3+}$ ions have approximately octahedral oxygen coordination. Thus the ground state of this ion is a double-degenerated ${ }^{5} E$ state. The degeneration could mainly be removed due to cooperative Jahn-Teller effect as in lanthanum manganite, ${ }^{24}$ but the strong distortion of the crystal structure may affect the orbital structure as well. ${ }^{22,23}$ A wave function of the ground state of each manganese ion $n$ is a linear combination of eigenfunctions $|\theta\rangle_{n},|\varepsilon\rangle_{n}$ of double-degenerated ${ }^{5} E$ ground state, used in Ref. 25:

$$
\psi_{n}=\sin \frac{\Phi_{n}}{2}|\theta\rangle_{n}+\cos \frac{\Phi_{n}}{2}|\varepsilon\rangle_{n},
$$

where $\Phi_{n}$ is a mixing angle of orbital functions.

TABLE I. The $C 2 / c$ crystal structure parameters in monoclinic reference frame according to Ref. 11 .

\begin{tabular}{llll}
\hline \hline Ion type & \multicolumn{1}{c}{$x$} & \multicolumn{1}{c}{$y$} & \multicolumn{1}{c}{$z$} \\
\hline Bi $(8 f)$ & 0.1375 & 0.2114 & 0.1269 \\
Mn1 $(4 e)$ & 0.0 & 0.219 & 0.75 \\
Mn2 $(4 d)$ & 0.25 & 0.25 & 0.5 \\
O1 $(8 f)$ & 0.097 & 0.173 & 0.578 \\
O2 $(8 f)$ & 0.145 & 0.568 & 0.366 \\
O3 $(8 f)$ & 0.356 & 0.547 & 0.166 \\
\hline \hline
\end{tabular}

The ground state on $\mathrm{Mn}^{3+}$ ion is described by the manyelectron configuration $\left[\left(t_{2 g}{ }^{3}\right)^{4} A_{2}, e_{g}\right]^{5} E$. Thus the many-body eigenfunctions of the ground state are determined as $d^{4}$ Slater determinants, depending of single-electron $\tilde{\xi}, \tilde{\eta}, \tilde{\zeta} t_{2 g}$ states and $\tilde{\theta}, \tilde{\varepsilon} e_{g}$ states:

$$
|\theta\rangle_{n}=-|\tilde{\xi} \tilde{\eta} \tilde{\zeta} \tilde{\varepsilon}|_{n}, \quad|\varepsilon\rangle_{n}=|\tilde{\xi} \tilde{\eta} \tilde{\zeta} \tilde{\theta}|_{n} .
$$

The corresponding wave function (1) of the orbital ground state may be rewritten as

$$
\psi_{n}=|\tilde{\xi} \tilde{\eta} \tilde{\zeta} \chi|_{n}
$$

where the single-electron wave function $\chi_{n}$ depends upon the eigenfunction of $n$th $e_{g}$ electron $|\tilde{\theta}\rangle_{n},|\tilde{\varepsilon}\rangle_{n}$

$$
\chi_{n}=\cos \frac{\Phi_{n}}{2}|\tilde{\theta}\rangle_{n}-\sin \frac{\Phi_{n}}{2}|\tilde{\varepsilon}\rangle_{n}
$$

This way of describing wave functions is used in many papers, for example, in Ref. 22.

The orbital structure of manganese sublattice is formed by vibronic interactions, which consist of three parts:

$$
H_{\mathrm{vib}}=H_{\mathrm{lin}}+H_{Q Q}+H_{R} .
$$

The main part of vibronic interaction is the linear electron-lattice interaction due to cooperative Jahn-Teller effect:

$$
H_{\mathrm{lin}}=V_{e} \sum_{n}\left(Q_{\theta n} X_{\theta n}+Q_{\varepsilon n} X_{\varepsilon n}\right)
$$

where

$$
X_{\theta n}=\left(\begin{array}{cc}
-1 & 0 \\
0 & 1
\end{array}\right), \quad X_{\varepsilon n}=\left(\begin{array}{ll}
0 & 1 \\
1 & 0
\end{array}\right)
$$

are orbital operators build on wave functions (1) and (2), $Q_{\theta n}$ and $Q_{\varepsilon n}$ are cooperative symmetrized $e_{g}$ distortions of oxygen ions in $n$th $\mathrm{MnO}_{6}$ octahedron (the calculation and visualization is shown in Appendix), and $V_{e}$ is a parameter of linear vibronic interaction.

In lanthanum manganite, the linear vibronic interaction was sufficient for the formation of orbital structure. ${ }^{24}$ The orbital structure could be calculated, using the symmetrized distortion of nearest-neighbor oxygen coordination:

$$
\cos \Phi_{n}^{0}=\frac{Q_{\theta n}}{\rho_{n}} ; \quad \sin \Phi_{n}^{0}=\frac{Q_{\varepsilon n}}{\rho_{n}} ; \quad \rho_{n}=\sqrt{Q_{\theta n}^{2}+Q_{\varepsilon n}^{2}} .
$$

In the strongly distorted $\mathrm{BiMnO}_{3}$ crystal, the other types of vibronic interaction could affect the orbital structure. We suppose that the $t_{1 g}$-type distortions of oxygen octahedra may change the proportion of $|\theta\rangle_{n},|\varepsilon\rangle_{n}$ wave functions as in lanthanum titanate. ${ }^{23}$ Also, the anharmonic interaction due to $e_{g}$ distortions plays a role in the orbital state, ${ }^{26}$

$$
\begin{aligned}
H_{Q Q}= & V_{b} \sum_{n}\left[\left(2 Q_{z n}^{2}-Q_{x n}^{2}-Q_{y n}^{2}\right) X_{\theta n}\right. \\
& \left.+\sqrt{3}\left(Q_{x n}^{2}-Q_{y n}^{2}\right) X_{\varepsilon n}\right] \\
& +N_{e} \sum_{n}\left[\left(Q_{\varepsilon n}^{2}-Q_{\theta n}^{2}\right) X_{\theta n}+2 Q_{\theta n} Q_{\varepsilon n} X_{\varepsilon n}\right],
\end{aligned}
$$


where $Q_{\theta n}$ and $Q_{\varepsilon n}$ are cooperative symmetrized $e_{g}$ distortions of oxygen ions in nth $\mathrm{MnO}_{6}$ octahedron, $Q_{x n}, Q_{\mathrm{y} n}$, and $Q_{\mathrm{zn}}$ are cooperative symmetrized $t_{1 g}$ distortions of oxygen ions in nth $\mathrm{MnO}_{6}$ octahedron (the calculation and visualization is shown in Appendix), $V_{b}$ and $N_{e}$ are vibronic constants.

$\mathrm{The} \mathrm{Bi}^{3+}$ ions are strongly polarized and shifted from the symmetric position. $\mathrm{A} \mathrm{Bi}^{3+}$ dipole moment affects the orbital state of $\mathrm{Mn}^{3+}$ ion. This effect could be taken into account in the vibronic constant. The bismuth sublattice symmetrized shifts are taken into account explicitly. Thus the effect of secondneighbor coordination of manganese ions must be taken into account like in Ref. 23,

$$
H_{R}=V_{e}^{R} \sum_{n}\left(Q_{\theta n}^{R} X_{\theta n}+Q_{\varepsilon n}^{R} X_{\varepsilon n}\right),
$$

where $Q^{R}{ }_{\theta n}$ and $Q^{R}{ }_{\varepsilon n}$ are cooperative symmetrized $e_{g}$ distortions of bismuth ions of nth manganese ion (the calculation and visualization is shown in Appendix), $V^{R}{ }_{e}$ is the secondneighbor vibronic constant.

The contributions of Eqs. (9) and (10) must be smaller than that of Eq. (7), and they could change the general form of the ground state (1). The changes in $\Phi_{n}$ angles could be described as

$$
\cos \Phi_{n}=\frac{\tilde{Q}_{\theta n}}{\tilde{\rho}_{n}}, \quad \sin \Phi_{n}=\frac{\tilde{Q}_{\varepsilon n}}{\tilde{\rho}_{n}},
$$

where

$$
\begin{gathered}
\tilde{Q}_{\theta n}=Q_{\theta n}+\frac{V_{b}}{V_{e}}\left(2 Q_{z n}^{2}-Q_{x n}^{2}-Q_{y n}^{2}\right) \\
+\frac{N_{e}}{V_{e}}\left(Q_{\varepsilon n}^{2}-Q_{\theta n}^{2}\right)+\frac{V_{e}^{R}}{V_{e}} Q_{\theta n}^{R}, \\
\tilde{Q}_{\varepsilon n}=Q_{\varepsilon n}+\frac{\sqrt{3} V_{b}}{V_{e}}\left(Q_{x n}^{2}-Q_{y n}^{2}\right)+\frac{2 N_{e}}{V_{e}} Q_{\theta n} Q_{\varepsilon n}+\frac{V_{e}^{R}}{V_{e}} Q_{\varepsilon n}^{R}, \\
\tilde{\rho}_{n}=\sqrt{\tilde{Q}_{\theta n}^{2}+\tilde{Q}_{\varepsilon n}^{2}} .
\end{gathered}
$$

The constants of vibronic interaction in Eqs. (5)-(10) are not yet defined, but we can discuss their influence on the orbital structure of $\mathrm{BiMnO}_{3}$. Using the crystal structure data of Ref. 11, one can find the symmetrized distortions of oxygen and bismuth coordination of each manganese ion. The values of distortions are shown in Table II. There are four types of manganese ions distinguished by values and signs of symmetrized distortions. They are numbered in Fig. 1. The details of symmetrized distortion types and calculations are given in Appendix.

The orbital structure within the framework of linear vibronic interactions, presented by Eqs. (6)-(8), is

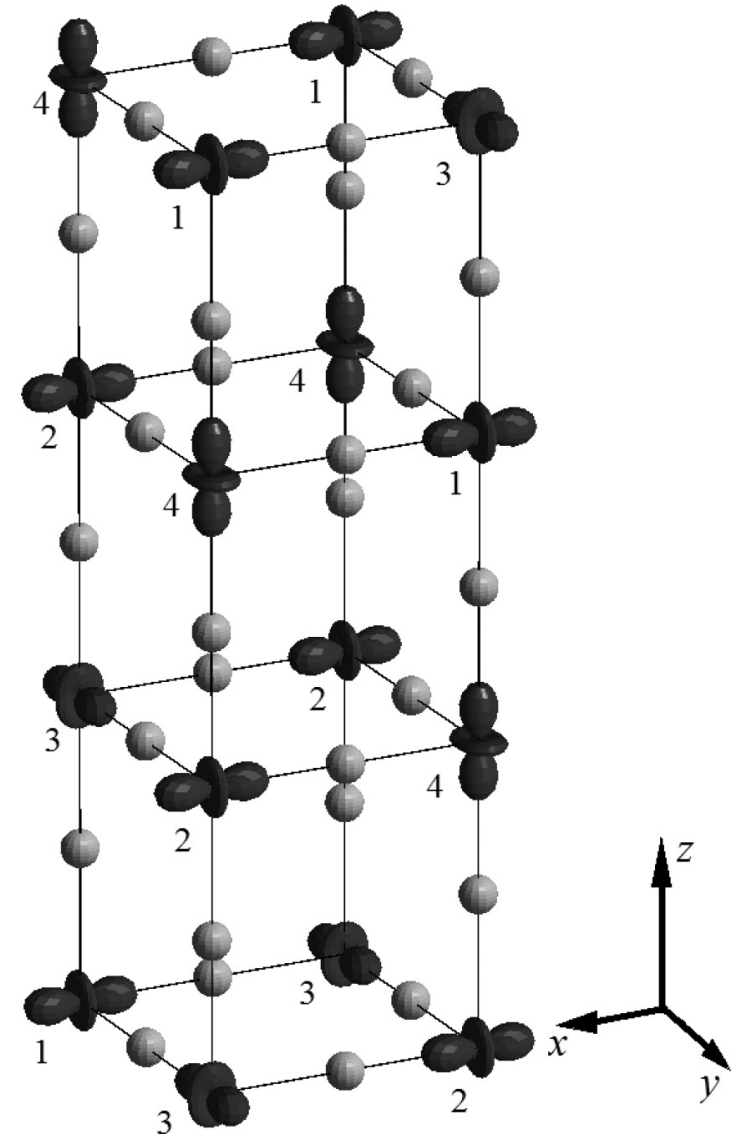

FIG. 1. Orbital structure of $\mathrm{BiMnO}_{3}$ [approximated by Eqs. (6)(8)]. The oxygen ions are denoted as spheres, the manganese ions are draws as orbital distributions. The pseudoperovskite reference frame is drawn. For perception convenience, the deflected vertical Mn-Mn bonding line coincides with pseudoperovskite $z$ axis. The true relation between pseudoperovskite $z$ axis and $\mathrm{Mn}-\mathrm{Mn}$ bond line see on Fig. 5 in Appendix.

approximately drawn in Fig. 1. The numerical values of orbital-mixing angles $\Phi_{n}$ are given in Table II.

The orbital structure of $\mathrm{BiMnO}_{3}$ is considerably different when compared with the lanthanum manganite case. ${ }^{24}$ The orbital mixing angles differ at various ions not only by the sign but also by value. This kind of orbital structure leads to qualitatively other types of magnetic ordering.

The influence of terms (8) and (9) upon the orbital structure will be discussed in another part of this paper. Because the calculations of $V_{b} / V_{e}, N_{e} / V_{e}$, and $V^{R} e_{e} / V_{e}$ relations for $\mathrm{BiMnO}_{3}$ are not yet carried out, one needs some reasonable considerations to evaluate them in order to find the exact orbital structure. As a topic for discussion one could take a magnetic structure of the compound.

TABLE II. Orbital structure mixing angles of symmetrized distortions.

\begin{tabular}{lrrrrrrrr}
\hline \hline Position and number of $\mathrm{Mn}^{3+}$ & $Q_{\varepsilon n}, \AA$ & $Q_{\theta n}, \AA$ & $Q_{x n}, \AA$ & $Q_{y n}, \AA$ & $Q_{z n}, \AA$ & $Q^{R_{\theta n},}, \AA$ & $Q^{R_{\varepsilon n}, \AA}$ & $\Phi_{n}^{0}$, deg \\
\hline $4 e, 1$ & 0.26 & -0.15 & 0.14 & 0.73 & -0.58 & -0.11 & 0.12 & 119 \\
$4 e, 2$ & 0.26 & -0.15 & 0.14 & 0.73 & -0.58 & -0.11 & 0.12 & 120 \\
$4 d, 3$ & -0.37 & -0.16 & -0.43 & -1.02 & 0.28 & -0.03 & 0.28 & -113 \\
$4 d, 4$ & -0.06 & 0.41 & 0.75 & -0.16 & 1.14 & -0.17 & 0.03 & -8 \\
\hline \hline
\end{tabular}




\section{SUPEREXCHANGE INTERACTION AND FRUSTRATED MAGNETIC STRUCTURE}

Despite the complicated orbital structure, the magnetic ordering is of the simple ferromagnetic arrangement according to experimental investigations of Refs. 6, 8-11. The superexchange interaction could be described, using the orbital operators approach. ${ }^{25}$ The Hamiltonian of superexchange interaction within $\mathrm{Mn}_{2} \mathrm{O}_{11}$ cluster arranged along the pseudoperovskite $z$ direction of undistorted crystal is

$\hat{H}_{\mathrm{ex}}^{z}=\frac{J_{0} \cos ^{2} \varphi_{n m}}{r_{n m}^{10}}\left[1+\alpha\left(X_{n \theta}+X_{m \theta}\right)+\beta X_{n \theta} X_{m \theta}\right]\left(\mathbf{S}_{n} \cdot \mathbf{S}_{m}\right)$,

where $X_{\theta n}$ and $X_{\varepsilon n}$ are the orbital operators (7), $J_{0}=$ $1.69 \times 10^{4} \mathrm{~K}^{10}, \alpha=1.0, \beta=4.5$ from Ref. $25, r_{n m}$ is the average $\mathrm{Mn}-\mathrm{O}$ distance in the interacting pair of ions $n$ and $m$, $\varphi_{n m}$ is the bonding angle $\mathrm{Mn}-\mathrm{O}-\mathrm{Mn}$ from the experimental studies of Ref. 11 . If the $\mathrm{Mn}_{2} \mathrm{O}_{11}$ cluster is arranged along the pseudoperovskite $x$ or $y$ direction, the superexchange interaction is 25

$$
\begin{aligned}
\hat{H}_{\mathrm{ex}}^{x(y)}= & \frac{J_{0} \cos ^{2} \varphi_{n m}}{r_{n m}^{10}}\left[1-\frac{\alpha}{2}\left(X_{n \theta} \pm \sqrt{3} X_{n \varepsilon}+X_{m \theta} \pm \sqrt{3} X_{m \varepsilon}\right)\right. \\
& \left.+\frac{\beta}{4}\left(X_{n \theta} \pm \sqrt{3} X_{n \varepsilon}\right)\left(X_{n \theta} \pm \sqrt{3} X_{n \varepsilon}\right)\right]\left(\mathbf{S}_{n} \cdot \mathbf{S}_{m}\right) .
\end{aligned}
$$

Averaging the values of Hamiltonians (15) and (16), calculated on wave function (1), leads to a general superexchange interaction Hamiltonian

$$
H_{\mathrm{ex}}=\sum_{\alpha, n m} J_{\alpha}^{n m}\left(\Phi_{m} ; \Phi_{n}\right)\left(\mathbf{S}_{n} \cdot \mathbf{S}_{m}\right),
$$

where the exchange parameters could be found as functions of orbital mixing angles of each pair $\Phi_{n}, \Phi_{m}$, directed along pseudoperovskite axes $\alpha=x, y, z$ :

$$
J_{\alpha}^{n m}\left(\Phi_{n}, \Phi_{m}\right)=\frac{J_{0} \cos ^{2} \varphi_{n m}}{r_{n m}^{10}} F_{\alpha}\left(\Phi_{n}, \Phi_{m}\right)
$$

and

$$
\begin{aligned}
F_{z}\left(\Phi_{n}, \Phi_{m}\right)= & 1+\alpha\left(\cos \Phi_{n}+\cos \Phi_{m}\right)+\beta \cos \Phi_{n} \cos \Phi_{m} \\
F_{x, y}\left(\Phi_{n}, \Phi_{m}\right)= & 1-\frac{\alpha}{2}\left(\cos \Phi_{n} \pm \sqrt{3} \sin \Phi_{n}+\cos \Phi_{m}\right. \\
& \left. \pm \sqrt{3} \sin \Phi_{m}\right)+\frac{\beta}{4}\left(\cos \Phi_{n} \pm \sqrt{3} \sin \Phi_{n}\right) \\
& \times\left(\cos \Phi_{m} \pm \sqrt{3} \sin \Phi_{m}\right) .
\end{aligned}
$$

Within the framework of the linear vibronic interaction model and using the experimental crystal structure from Ref. 11, the superexchange parameters are calculated. The results are presented in Table III.

The superexchange interactions divide the magnetic subsystem of $\mathrm{BiMnO}_{3}$ crystal into $O y z$ planes of pseudoperovskite reference frame. Within these planes, one could find ferromagnetic zigzag chains, coupled antiferromagnetically, as shown in Fig. 2. The interplane superexchange interaction is ferromagnetic, but the chains in the neighboring planes are shifted along the $y$ axis relative to each other. This shift, together with the antiferromagnetic interaction, leads to a competition between exchange interactions in different pairs.
TABLE III. The superexchange parameters of $\mathrm{BiMnO}_{3}$ [model set by Eqs. (1), (6)-(8), (18), and (19)]. The negative parameters denote FM type of interaction and positive ones correspond to AFM interaction.

\begin{tabular}{llrr}
\hline \hline & \multicolumn{3}{c}{ Superexchange interaction, $\mathrm{K}$} \\
\cline { 2 - 4 } Pairs of $\mathrm{Mn}^{3+}$ ions & $J_{x}$ & $J_{y}$ & $J_{z}$ \\
\hline $1-3$ & -12 & -9 & 13 \\
$1-4$ & -13 & 13 & -8 \\
$2-3$ & -12 & -9 & 13 \\
$2-4$ & -12 & 13 & -8 \\
\hline \hline
\end{tabular}

There are some magnetically frustrated cells with $x y$ or $x z$ orientation, which have three ferromagnetic bonds and one antiferromagnetic one. Taking into account the near values of different kinds of superexchange interaction, we come to a conclusion that the magnetic structure could not be simply determined as ferromagnetic. There are three types of magnetic structures that could be formed in the crystal with similar orbital structures like on Fig. 1 and superexchange signs like on Fig. 2: $F$-type ferromagnetic, $A$-type antiferromagnetic (with FM $O x y$ planes), and $C$-type antiferromagnetic (with FM $O x$ chains).

The exchange parameters shown in Table III within the framework of linear nearest-neighbor vibronic interaction (6) describe the AFM $A$-type structure. A variation of phenomenological $J_{0}, \alpha$, and $\beta$ constants of orbitally dependent exchange interaction (18) and (19) within the range of $20 \%$ near values of Ref. 25 is not enough to shift the magnetic ground state from AFM order to FM one. Thus we conclude that the vibronic Hamiltonian must be more complicated than it is supposed in Eq. (5).

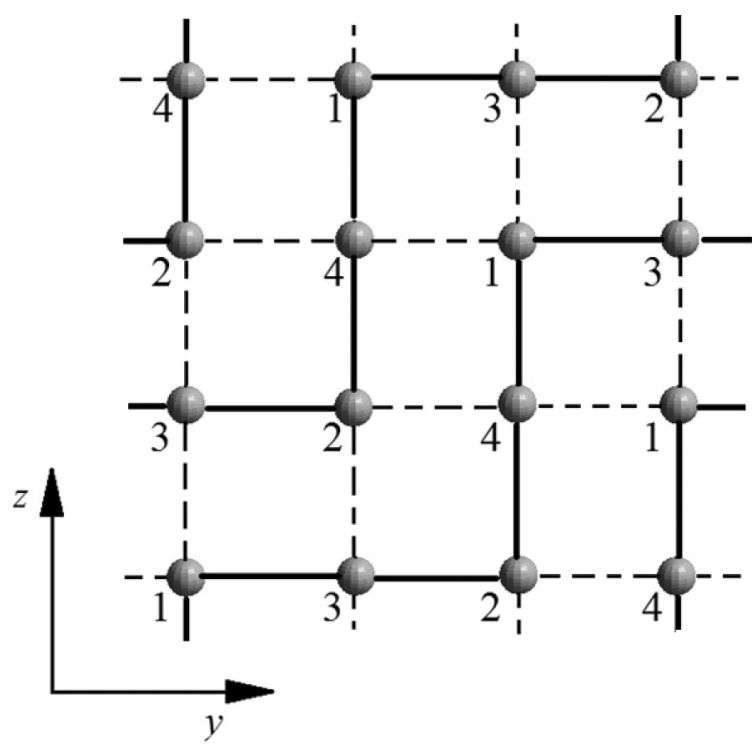

FIG. 2. Ferromagnetic zigzag chains (solid lines) in pseudoperovskite $O y z$ plane. Dashed lines denote antiferromagnetic bonds. Spheres denote magnetic $\mathrm{Mn}^{3+}$ ions. 


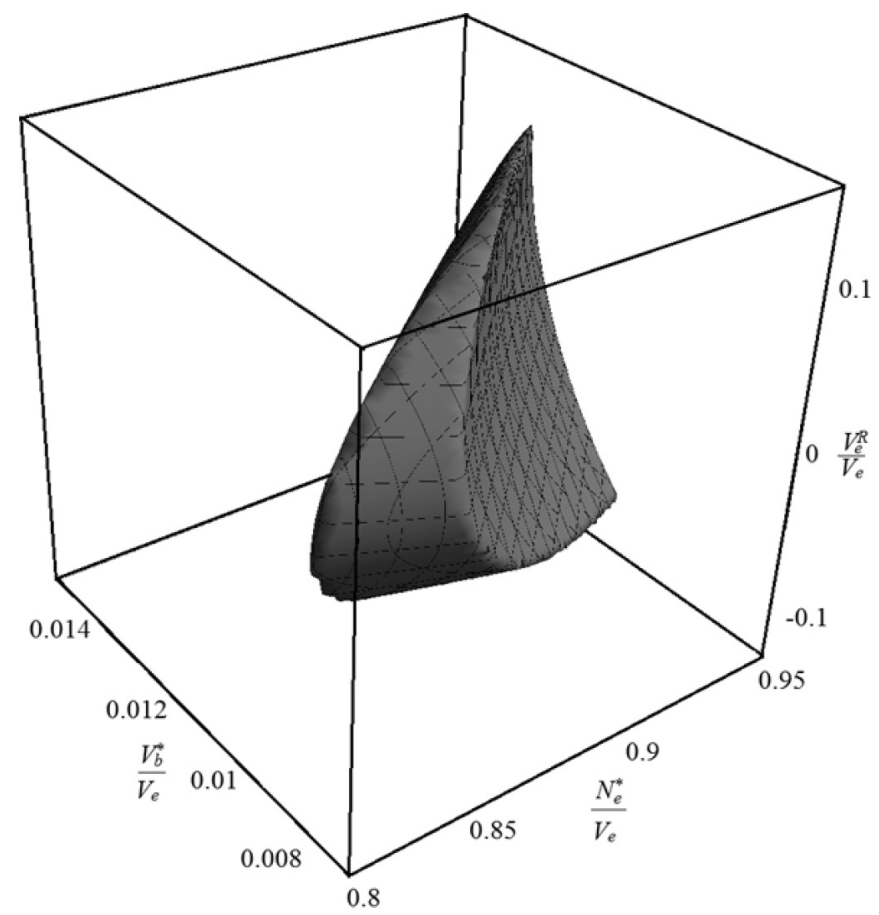

FIG. 3. The diagram of $V^{*}{ }_{b} / V_{e}, N^{*}{ }_{e} / V_{e}$, and $V^{R}{ }_{e} / V_{e}$ relations.

\section{RESULTS AND DISCUSSION}

In order to determine a possibility of FM structure depending upon $V_{b} / V_{e}, N_{e} / V_{e}$, and $V_{e}^{R} / V_{e}$ relations, the following considerations must be taken into account: (1) the experimental value of Curie temperature is $T_{C}=100 \mathrm{~K} .{ }^{11}$ The superexchange parameters must give an adequate estimation of Curie temperature. (2) The energies of $C$ - and $A$-type antiferromagnetic structures must be higher than the $F$-type one. (3) The terms of additional contributions in orbital structure must be at least at a lower absolute value than the main term due to linear vibronic interaction.

The comparison of constants having different units is inconvenient. In order to bring $V_{b} / V_{e}, N_{e} / V_{e}$ relations in dimensionless form, the $N_{e}$ and $V_{b}$ could be multiplied by Bohr radius $a_{0}=0.529 \AA$. This value is typical for ionic displacements and is of the same order with symmetrized distortions in the crystal. New constants $V_{b}{ }^{*}=\mathrm{V}_{b} a_{0}$ and $N_{e}{ }^{*}=\mathrm{N}_{e} a_{0}$ produce dimensionless relations $V_{b}{ }^{*} / V_{e}, N_{e}{ }^{*} / V_{e}$.

In Fig. 3, the region of $V^{*}{ }_{b} / V_{e}, N^{*}{ }_{e} / V_{e}$, and $V^{R}{ }_{e} / V_{e}$ relations satisfying conditions $1-3$ is drawn. The Curie temperature is taken approximately $T_{C} \approx 100 \pm 5 \mathrm{~K}$ within the framework of the mean-field approximation. The diagram shows that the crucial role of forming ferromagnetism belongs to the anharmonic term of $e_{g}$ distortions [the second summand of Eq. (9)]. The $N_{e}$ constant must be sufficiently large. The modified orbital structure generally remains of the same form as in Fig. 1. The forms of orbitals are noticeably changed only in manganese ions of third position from $d_{3 y^{2}-r^{2}}$ to $d_{y^{2}-z^{2}}$.

The exchange parameters, being approximately of the same magnitude according to Table III, became more anisotropic. The largest values belong to the $J_{x}$ parameters $(\geqslant 25 \mathrm{~K})$, and the interactions within the $O y z$ plane must be weaker $(\sim 6 \mathrm{~K})$. The basic feature of the interactions is present, i.e., the signs of superexchange parameters remain the same. Examples of possible structures and the corresponding set of superexchange parameters are shown in Table IV and Fig. 4.

The shown orbital structure is the closest to the JahnTeller one, but it is not the only structure determined by the diagram in Fig. 3. The marginal regions of vibronic constants diagrammed in Fig. 3 provide the drastic change of superexchange parameters relation, which could not to be taken into account.

The values of vibronic constants we could compare with the $a b$ initio calculations of Ref. 23 for $\mathrm{LaTiO}_{3}: V^{*}{ }_{b} / V_{e}=$ $-0.02, V_{e}^{R} / V_{e}=0.15$. The values in our estimation are of the same order. The value of $N_{e}$ constant is the largest among the additional vibronic interactions. It could be compared with earlier MO LKAO calculations of the same interaction parameters in $\mathrm{KCuF}_{3}$ crystal ${ }^{26}$ where the relation is $N^{*}{ }_{e} / V_{e}=$ 0.2 and with $\mathrm{RbMnF}_{3}{ }^{27}$ where for an exited manganese state the relation is $N^{*}{ }_{e} / V_{e}=0.73$. The comparison shows that the large value used for the calculation in the example in Table III, $N^{*}{ }_{e} / V_{e}=0.82$, could take place in the case of manganese ions. Thus the adjustment of orbital structure changes the parameters of superexchange interaction twice in the most pairs. The FM interaction in $O x$ pseudoperovskite direction plays the main role in FM ordering.

The values of superexchange interaction were calculated by Soloviev et al..$^{7,18}$ They found the magnitude of FM interaction as $\left|J_{N N}\right| \sim 60 \mathrm{~K}$. It is larger than our values and does not provide the Curie temperature of the experimental value $T_{C}=$ $100 \mathrm{~K}$. The antiferromagnetic interaction parameters of Refs. 7 and 18 are of the same order. These investigations use the next-nearest neighbor antiferromagnetic exchange interaction to explain the FE state via a canted magnetic structure, but there are no experimental studies of single crystals confirming FE properties of $\mathrm{BiMnO}_{3}$. Thus using the long-range exchange interaction of the large values $(\sim 10 \mathrm{~K})$ seems to be not sufficiently argued.

TABLE IV. Example of possible orbital structure of $\mathrm{BiMnO}_{3}$ due to anharmonic and next-nearest neighbor vibronic interactions [Eqs. (9)-(14)].

\begin{tabular}{lcrrr}
\hline \hline & & & \multicolumn{2}{c}{ Superexchange interaction, K } \\
\cline { 3 - 5 } Vibronic interaction parameters & Orbital structure angles, degrees & Pair of $\mathrm{Mn}^{3+}$ ions & $J_{x}$ & $J_{y}$ \\
\hline$V^{*}{ }_{b} / V_{e}=0.008$ & $\Phi_{1}=120$ & $1-3$ & -24 & -7 \\
$N^{*}{ }_{e} / V_{e}=0.82$ & $\Phi_{2}=120$ & $1-4$ & -26 & -7 \\
$V^{R}{ }_{e} / V_{e}=0.05$ & $\Phi_{3}=-92$ & $2-3$ & -25 & -6 \\
& $\Phi_{4}=-35$ & $2-4$ & -24 & 6 \\
\hline \hline
\end{tabular}




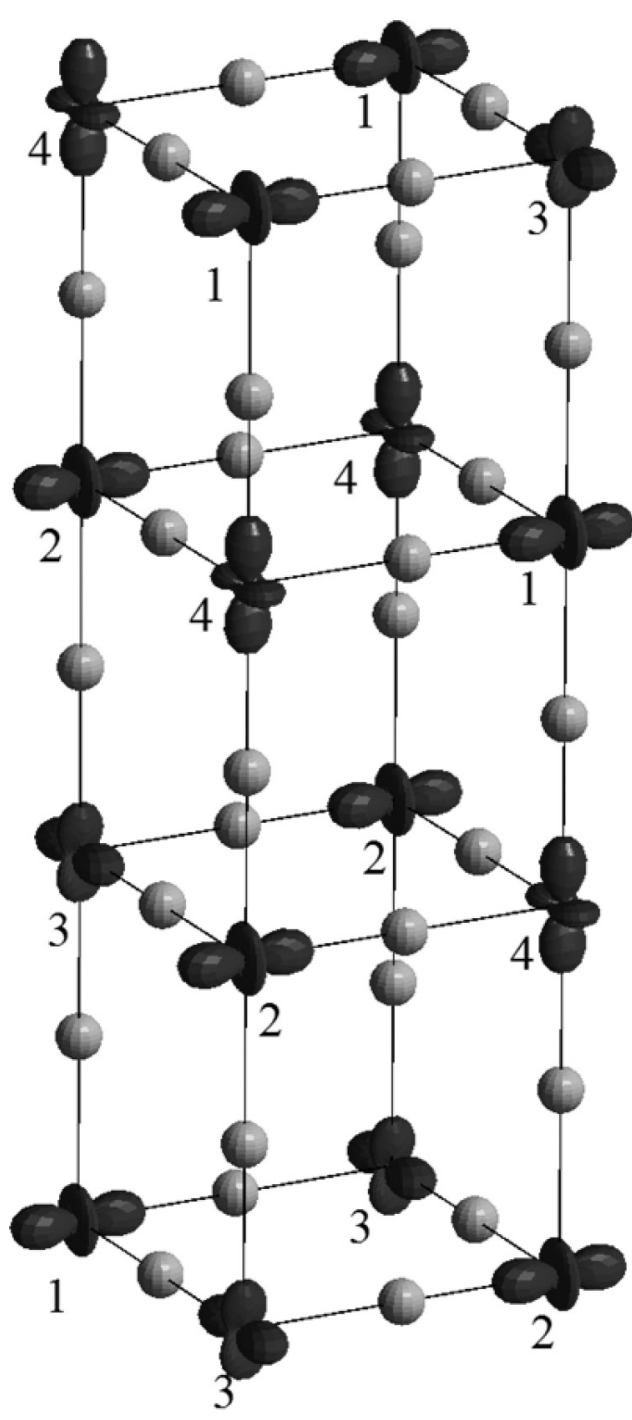

FIG. 4. The example of orbital structure giving FM ordering.

In Fig. 2, one could recognize the same zigzag ordering of $\mathrm{CO}$ manganite $\mathrm{La}_{0.5} \mathrm{Ca}_{0.5} \mathrm{MnO}_{3} .{ }^{24}$ However, $\mathrm{CE}$ magnetic structure like in $\mathrm{CO}$ manganites is impossible in this case, because the pseudoperovskite $O x y$ planes with FM interaction between each other are ordered with a shift. This shift causes a competition between superexchange interactions.

The reasons explaining the modified vibronic interaction could be used for the explanation of magnetic ordering in the frustrated $\mathrm{CO}$ manganite $\mathrm{La}_{0.333} \mathrm{Ca}_{0.667} \mathrm{MnO}_{3}{ }^{28}$ The orbital structure due to linear vibronic interaction must be corrected by changing the orbital mixing angle at $5^{\circ}$ in order to describe the magnetic unit cell. The reason for the correction could be the additional terms of vibronic Hamiltonian.

The effect of anharmonicity in regular manganites $R \mathrm{MnO}_{3}$ ( $R=\mathrm{La}, \mathrm{Nd}, \mathrm{Pr}$ ) also could be taken into account, but the adjusted orbital structure produces no change in signs of superexchange interaction parameters. Thus the magnetic structure without frustration remains the same in spite of the difference between the adjusted orbital structure and ordering due to linear vibronic interaction.

\section{CONCLUSIONS}

Thus the advanced model of the vibronic interaction in Jahn-Teller crystals is proposed. This model could be applied to orbital structure description in the case of magnetic frustration, in order to explain the complicated magnetic structures. The necessity to expand the vibronic Hamiltonian of magnetically frustrated compounds is proved by the fact that the weak interaction changing orbital structure affects the superexchange interaction equilibrium, moving it to another type of magnetic structure. In the case of bismuth manganite, the equilibrium antiferromagnetic state changes to a ferromagnetic one due to the orbital anharmonicity and next-nearest neighbor vibronic interaction. The Curie temperature of $\mathrm{BiMnO}_{3}$ is also explained by this model.

\section{ACKNOWLEDGMENT}

The authors are grateful to D. P. Kozlenko (Frank Laboratory of Neutron Physics, JINR, Dubna, Russia) for the idea of the investigation.

\section{APPENDIX: SYMMETRIZED DISTORTION CALCULATIONS}

The main problem of the symmetrized distortion calculation is the undistorted axes choice. In the lanthanum manganite ${ }^{24,28}$ and titanate ${ }^{23}$ crystals, there were the $M \mathrm{O}_{6}$ octahedra $(M=$ $\mathrm{Mn}^{3+}, \mathrm{Ti}^{3+} \mathrm{JT}$ ions) with orthogonal $M-\mathrm{O}$ axes. Thus one could make a projection on the undistorted axes taking into account the octahedron rotation. In $\mathrm{BiMnO}_{3}$ case, there is a non-Carthesian local reference frame, associated with $\mathrm{MnO}_{6}$ octahedron axes.

In order to choose undistorted octahedron axes, we assume pseudoperovskite $x$ and $y$ axes directed along orthogonal Mn1-Mn3 bonds, and the $z$ axis is chosen to be directed perpendicular to $O x y$ plane along one of Mn2-Mn4 bonds. The Mn1-Mn3 nonorthogonal bond is deflected from this axis (like $z$ axis in Fig. 2). In neighboring octahedron, the Mn2-Mn4 bond is deflected from $x$ axis. The position coordinates and the undistorned octahedron axes have to be transferred to the Carthesian reference frame. The orts of this reference frame, associated with monoclinic unit cell, are

$$
\mathbf{i}_{m}=\frac{\mathbf{a}}{a}, \quad \mathbf{j}_{m}=\frac{\mathbf{b}}{b}, \quad \mathbf{k}_{m}=\frac{1}{\sin \beta}\left(\frac{\mathbf{c}}{c}-\frac{\mathbf{a}}{a} \cos \beta\right),
$$

where $a, b, c$ are lattice constants of $\mathrm{BiMnO}_{3}$ and $\mathbf{a}, \mathbf{b}, \mathbf{c}$ are vectors of $\mathrm{BiMnO}_{3}$ unit cell.

Within the reference frame (A1), the undistorted octahedron axes are directed along the perovskite orts:

$$
\begin{aligned}
\mathbf{i} & =(-0.82 ;-0.04 ; 0.58), \\
\mathbf{j} & =(-0.37 ; 0.73 ;-0.57), \\
\mathbf{k} & =(-0.40 ; 0.68 ; 0.61)
\end{aligned}
$$

A correlation between octahedron axes is drawn in Fig. 5. The types and expressions of symmetrized distortions coordinates are compiled from Ref. 23.

The $e_{g}$-type symmetrized distortions of each $\mathrm{MnO}_{6}$ octahedron are drawn in Fig. 6 (number $n$ of manganese ion is 


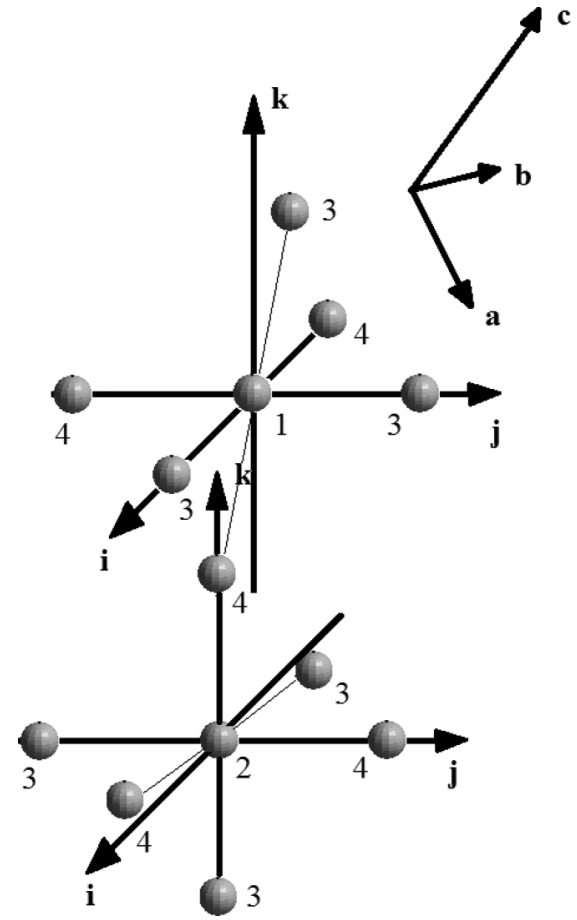

FIG. 5. Pseudoperovskite reference (i, $\mathbf{j}, \mathbf{k})$ frame compared with monoklinic reference frame $(\mathbf{a}, \mathbf{b}, \mathbf{c})$. Numbers denote manganese position according Table II.

omitted):

$$
\begin{aligned}
Q_{\theta}= & {\left[-\left(\mathbf{r}_{\mathrm{MnO}_{1}}-\mathbf{r}_{1}\right) \cdot \mathbf{i}-\left(\mathbf{r}_{\mathrm{MnO}_{2}}-\mathbf{r}_{2}\right) \cdot \mathbf{j}\right.} \\
& +2\left(\mathbf{r}_{\mathrm{MnO}_{3}}-\mathbf{r}_{3}\right) \cdot \mathbf{k}+\left(\mathbf{r}_{\mathrm{MnO}_{5}}-\mathbf{r}_{5}\right) \cdot \mathbf{i} \\
& \left.+\left(\mathbf{r}_{\mathrm{MnO}_{4}}-\mathbf{r}_{4}\right) \cdot \mathbf{j}-2\left(\mathbf{r}_{\mathrm{MnO}_{6}}-\mathbf{r}_{6}\right) \cdot \mathbf{k}\right] / \sqrt{12}, \\
Q_{\varepsilon}= & {\left[\left(\mathbf{r}_{\mathrm{MnO}_{1}}-\mathbf{r}_{1}\right) \cdot \mathbf{i}-\left(\mathbf{r}_{\mathrm{MnO}_{2}}-\mathbf{r}_{2}\right) \cdot \mathbf{j}\right.} \\
& \left.-\left(\mathbf{r}_{\mathrm{MnO}_{5}}-\mathbf{r}_{5}\right) \cdot \mathbf{i}+\left(\mathbf{r}_{\mathrm{MnO}_{4}}-\mathbf{r}_{4}\right) \cdot \mathbf{j}\right] / 2,
\end{aligned}
$$

where $\mathbf{r}_{i}(i=1, \ldots, 6)$ are $\mathrm{Mn}-\mathrm{O}$ distances of undistorted octahedron, numbered according to Fig. 6,

$$
\begin{aligned}
& \mathbf{r}_{1}=r_{0} \mathbf{i}, \quad \mathbf{r}_{2}=r_{0} \mathbf{j}, \quad \mathbf{r}_{3}=r_{0} \mathbf{k}, \\
& \mathbf{r}_{4}=-r_{0} \mathbf{j}, \quad \mathbf{r}_{2}=-r_{0} \mathbf{i}, \quad \mathbf{r}_{3}=r_{0} \mathbf{k}, \\
& r_{0}=\frac{1}{6} \sum_{i=1}^{6}\left|\mathbf{r}_{\mathrm{MnO}_{i}}\right|,
\end{aligned}
$$
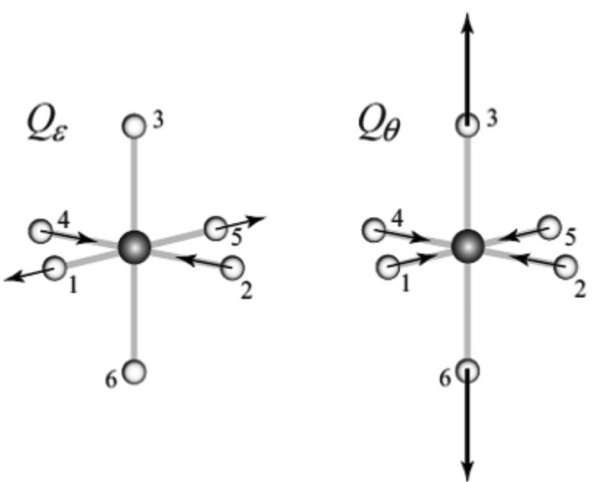

FIG. 6. Symmetrized $e_{g}$ distortion of $\mathrm{MnO}_{6}$ octahedron. Dark spheres denote manganese ions, light spheres denote oxygen ions, numbers indicate different oxygen positions.
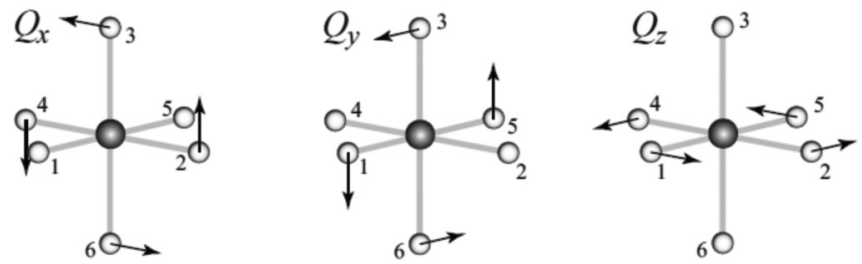

FIG. 7. Symmetrized $t_{1 g}$ distortion of $\mathrm{MnO}_{6}$ octahedron. Dark spheres denote manganese ions, light spheres denote oxygen ions, numbers indicate different oxygen positions.

and $\mathbf{r}_{\mathrm{MnO} i}(i=1, \ldots, 6)$ are the $\mathrm{Mn}-\mathrm{O}$ distances of distorted octahedron in the crystal.

The $t_{1 g}$-type symmetrized distortions of each $\mathrm{MnO}_{6}$ octahedron are drawn in Fig. 7 (number $n$ of manganese ion is omitted),

$$
\begin{aligned}
Q_{x}= & {\left[\left(\mathbf{r}_{\mathrm{MnO}_{2}}-\mathbf{r}_{2}\right) \cdot \mathbf{k}-\left(\mathbf{r}_{\mathrm{MnO}_{4}}-\mathbf{r}_{4}\right) \cdot \mathbf{k}\right.} \\
& \left.-\left(\mathbf{r}_{\mathrm{MnO}_{3}}-\mathbf{r}_{3}\right) \cdot \mathbf{j}+\left(\mathbf{r}_{\mathrm{MnO}_{6}}-\mathbf{r}_{6}\right) \cdot \mathbf{j}\right] / 2, \\
Q_{y}= & {\left[-\left(\mathbf{r}_{\mathrm{MnO}_{1}}-\mathbf{r}_{1}\right) \cdot \mathbf{k}+\left(\mathbf{r}_{\mathrm{MnO}_{5}}-\mathbf{r}_{5}\right) \cdot \mathbf{k}\right.} \\
& \left.-\left(\mathbf{r}_{\mathrm{MnO}_{3}}-\mathbf{r}_{3}\right) \cdot \mathbf{i}-\left(\mathbf{r}_{\mathrm{MnO}_{6}}-\mathbf{r}_{6}\right) \cdot \mathbf{i}\right] / 2, \\
Q_{z}= & {\left[\left(\mathbf{r}_{\mathrm{MnO}_{1}}-\mathbf{r}_{1}\right) \cdot \mathbf{j}-\left(\mathbf{r}_{\mathrm{MnO}_{5}}-\mathbf{r}_{5}\right) \cdot \mathbf{j}-\right.} \\
& \left.-\left(\mathbf{r}_{\mathrm{MnO}_{2}}-\mathbf{r}_{2}\right) \cdot \mathbf{i}+\left(\mathbf{r}_{\mathrm{MnO}_{4}}-\mathbf{r}_{4}\right) \cdot \mathbf{i}\right] / 2 .
\end{aligned}
$$

The $e_{g}$-type symmetrized distortions of next-nearest neighbor. The bismuth coordination of manganese ion is drawn in Fig. 8 (number $n$ is omitted);

$$
\begin{aligned}
Q_{\varepsilon}^{R}= & {\left[\left(\mathbf{r}_{\mathrm{MnBi}_{1}}-\mathbf{r}_{1}\right) \cdot(-\mathbf{i}+\mathbf{j})+\left(\mathbf{r}_{\mathrm{MnBi}_{2}}-\mathbf{r}_{2}\right) \cdot(\mathbf{i}+\mathbf{j})\right.} \\
& +\left(\mathbf{r}_{\mathrm{MnBi}_{3}}-\mathbf{r}_{3}\right) \cdot(\mathbf{i}-\mathbf{j})+\left(\mathbf{r}_{\mathrm{MnBi}_{4}}-\mathbf{r}_{4}\right) \cdot(-\mathbf{i}-\mathbf{j}) \\
& +\left(\mathbf{r}_{\mathrm{MnBi}_{5}}-\mathbf{r}_{5}\right) \cdot(-\mathbf{i}+\mathbf{j})+\left(\mathbf{r}_{\mathrm{MnBi}_{6}}-\mathbf{r}_{6}\right) \cdot(\mathbf{i}+\mathbf{j}) \\
& \left.+\left(\mathbf{r}_{\mathrm{MnBi}_{7}}-\mathbf{r}_{7}\right) \cdot(\mathbf{i}-\mathbf{j})+\left(\mathbf{r}_{\mathrm{MnBi}_{8}}-\mathbf{r}_{8}\right) \cdot(-\mathbf{i}-\mathbf{j})\right] / 4, \\
Q_{\theta}^{R}= & {\left[\left(\mathbf{r}_{\mathrm{MnBi}_{1}}-\mathbf{r}_{1}\right) \cdot(-\mathbf{i}-\mathbf{j}+2 \mathbf{k})\right.} \\
& +\left(\mathbf{r}_{\mathrm{MnBi}_{2}}-\mathbf{r}_{2}\right) \cdot(\mathbf{i}-\mathbf{j}+2 \mathbf{k}) \\
& +\left(\mathbf{r}_{\mathrm{MnBi}_{3}}-\mathbf{r}_{3}\right) \cdot(\mathbf{i}+\mathbf{j}+2 \mathbf{k}) \\
& +\left(\mathbf{r}_{\mathrm{MnBi}_{4}}-\mathbf{r}_{4}\right) \cdot(-\mathbf{i}+\mathbf{j}+2 \mathbf{k}) \\
& +\left(\mathbf{r}_{\mathrm{MnBi}_{5}}-\mathbf{r}_{5}\right) \cdot(-\mathbf{i}-\mathbf{j}-2 \mathbf{k}) \\
& +\left(\mathbf{r}_{\mathrm{MnBi}_{6}}-\mathbf{r}_{6}\right) \cdot(\mathbf{i}-\mathbf{j}-2 \mathbf{k})
\end{aligned}
$$

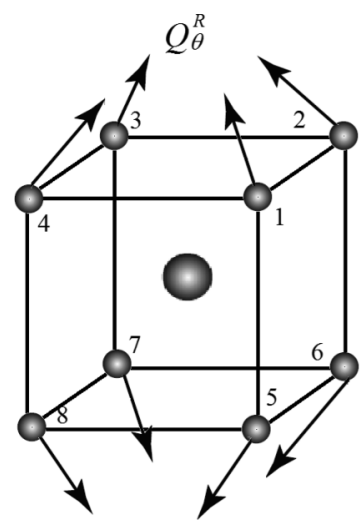

$Q_{\varepsilon}^{R}$

FIG. 8. Symmetrized $e_{g}$ distortion of $\mathrm{MnBi}_{8}$ cluster. Large spheres denote manganese ions, small spheres denote bismuth ions, numbers indicate different bismuth positions. 


$$
\begin{aligned}
& +\left(\mathbf{r}_{\mathrm{MnBi}_{7}}-\mathbf{r}_{7}\right) \cdot(\mathbf{i}+\mathbf{j}-2 \mathbf{k}) \\
& \left.+\left(\mathbf{r}_{\mathrm{MnBi}_{8}}-\mathbf{r}_{8}\right) \cdot(-\mathbf{i}+\mathbf{j}-2 \mathbf{k})\right] /(2 \sqrt{12}),
\end{aligned}
$$

where $\mathbf{r}_{i}(i=1, \ldots, 8)$ are $\mathrm{Mn}-\mathrm{Bi}$ distances of bismuth environment, numbered according to Fig. 8,

$$
\begin{aligned}
& \mathbf{r}_{1}=R_{0}(\mathbf{i}+\mathbf{j}+\mathbf{k}) / \sqrt{3}, \\
& \mathbf{r}_{2}=R_{0}(-\mathbf{i}+\mathbf{j}+\mathbf{k}) / \sqrt{3}, \\
& \mathbf{r}_{3}=R_{0}(-\mathbf{i}-\mathbf{j}+\mathbf{k}) / \sqrt{3}, \\
& \mathbf{r}_{4}=R_{0}(\mathbf{i}-\mathbf{j}+\mathbf{k}) / \sqrt{3},
\end{aligned}
$$

$$
\begin{aligned}
& \mathbf{r}_{5}=R_{0}(\mathbf{i}+\mathbf{j}-\mathbf{k}) / \sqrt{3}, \\
& \mathbf{r}_{6}=R_{0}(-\mathbf{i}+\mathbf{j}-\mathbf{k}) / \sqrt{3}, \\
& \mathbf{r}_{7}=R_{0}(-\mathbf{i}-\mathbf{j}-\mathbf{k}) / \sqrt{3}, \\
& \mathbf{r}_{8}=R_{0}(\mathbf{i}-\mathbf{j}-\mathbf{k}) / \sqrt{3}, \\
& R_{0}=\frac{1}{8} \sum_{i=1}^{8}\left|\mathbf{r}_{\mathrm{MnBi}_{i}}\right|,
\end{aligned}
$$

and $\mathbf{r}_{\mathrm{MnBi} i}(i=1, \ldots, 8)$ are $\mathrm{Mn}-\mathrm{Bi}$ distances of distorted crystal. *lyudmila.gonchar@usu.ru

${ }^{1}$ Y. Takahashi, S. Ishiwata, S. Miyahara, Y. Kaneko, N. Furukawa, Y. Taguchi, R. Shimano, and Y. Tokura, Phys. Rev. B 81, 100413(R) (2010).

${ }^{2}$ D. P. Kozlenko, A. A. Belik, A. V. Belushkin, E. V. Lukin, W. G. Marshall, B. N. Savenko, and E. Takayama-Muromachi, Phys. Rev. B 84, 094108 (2011).

${ }^{3}$ D. I. Khomski, J. Magn. Magn. Mater. 3061 (2006); Physics 2, 20 (2009).

${ }^{4}$ A. P. Pyatakov and A. K. Zvezdin, Usp. Phys. Nauk 182, 593 (2012) [Phys. Usp. 55, 557 (2012)].

${ }^{5}$ M. Grizalez, E. Martinez, J. Caicedo, J. Heiras, and P. Prieto, Microelectron. J. 39, 1308 (2008).

${ }^{6}$ A. Moreira dos Santos, S. Parashar, A. R. Raju, Y. S. Zhao, A. K Cheetham, and C. N. R Rao, Solid State Commun. 122, 49 (2002).

${ }^{7}$ I. V. Solovyev and Z. V. Pchelkina, Phys. Rev. B 82, 094425 (2010).

${ }^{8}$ T. Atou, H. Chiba, K. Ohoyama, Y. Yamaguchi, and Y. Syono, J. Solid State Chem. 145, 639 (1999).

${ }^{9}$ A. Moreira dos Santos, A. K. Cheetham, T. Atou, Y. Syono, Y. Yamaguchi, K. Ohoyama, H. Chiba, and C. N. R. Rao, Phys. Rev. B 66, 064425 (2002).

${ }^{10}$ A. A. Belik, S. Iikubo, T. Yokosawa, K. Kodama, N. Igawa, S. Shamoto, M. Azuma, M. Takano, K. Kimoto, Y. Matsui, and E. Takayama-Muromachi, J. Am. Chem. Soc. 129, 977 (2007); H. Yanga, Z. H. Chi, J. L. Jiang, W. J. Feng, Z. E. Caoa, T. Xian, C. Q. Jin, and R. C. Yu, J. Alloys Compd. 461, 1 (2008); A. A. Belik, K. Kodama, N. Igawa, S. Shamoto, K. Kosuda, and E. Takayama-Muromachi, J. Am. Chem. Soc. 132, 8137 (2010).

${ }^{11}$ D. P. Kozlenko, A. A. Belik, S. E. Kichanov, I. Mirebeau, D. V. Sheptyakov, Th. Strässle, O. L. Makarova, A. V. Belushkin, B. N. Savenko, and E. Takayama-Muromachi, Phys. Rev. B 82, 014401 (2010).

${ }^{12}$ E. Montanari, G. Calestani, L. Righi, E. Gilioli, F. Bolzoni, K. S. Knight, and P. G. Radaelli, Phys. Rev. B 75, 220101(R) (2007).

${ }^{13}$ T. Kimura, S. Kawamoto, I. Yamada, M. Azuma, M. Takano, and Y. Tokura, Phys. Rev. B 67, 180401(R) (2003).

${ }^{14}$ C. C. Chou, S. Taran, J. L. Her, C. P. Sun, C. L. Huang, H. Sakurai, A. A. Belik, E. Takayama-Muromachi, and H. D. Yang, Phys. Rev. B 78, 092404 (2008).
${ }^{15}$ C.-H. Yang, T. Y. Koo, S.-H. Lee, C. Song, K.-B. Lee, and Y. H. Jeong, Europhys. Lett. 74, 348 (2006); C.-H. Yang, J. Koo, C. Song, T. Y. Koo, K.-B. Lee, and Y. H. Jeong, Phys. Rev. B 73, 224112 (2006).

${ }^{16}$ D. W. Boukhvalov and I. V. Solovyev, Phys. Rev. B 82, 245101 (2010).

${ }^{17}$ J. M. Chen, S. C. Haw, J. M. Lee, S. A. Chen, K. T. Lu, S. W. Chen, M. J. Deng, Y. F. Liao, J. M. Lin, B. H. Chen, F. C. Chou, N. Hiraoka, H. Ishii, K. D. Tsuei, and E. Huang, Phys. Rev. B 86, 045103 (2012).

${ }^{18}$ I. V. Solovyev and Z. V. Pchelkina, New J. Phys. 10, 073021 (2008)

${ }^{19}$ C. C. Chou, C. L. Huang, S. Mukherjee, Q. Y. Chen, H. Sakurai, A. A. Belik, E. Takayama-Muromachi, and H. D. Yang, Phys. Rev. B 80, 184426 (2009).

${ }^{20}$ A. Castro, C. Correas, O. Pena, A. R. Landa-Canovas, M. Alguero, H. Amorın, M. Dolle, E. Vila, and T. Hungria, J. Mater. Chem. 22, 9928 (2012); A. A. Belik, Y. Matsushita, M. Tanaka, and E. Takayama-Muromachi, Inorg. Chem. 50, 7685 (2011).

${ }^{21}$ A. Kirste, M. Goiran, M. Respaud, J. Vanaken, J. M. Broto, H. Rakoto, M. von Ortenberg, C. Frontera, and J. L. Garcia-Munoz, Phys. Rev. B 67, 134413 (2003); C.-C. Yang, W.-H. Li, C.-M. Wu, C. H. C. Li, J. Sun, and J. W. Lynn, Inorg. Chem. 49, 3297 (2010).

${ }^{22}$ A. O. Sboychakov, K. I. Kugel, A. L. Rakhmanov, and D. I. Khomskii, Phys. Rev. B 83, 205123 (2011).

${ }^{23}$ A. A. Mozhegorov, A. V. Larin, A. E. Nikiforov, L. E. Gontchar, and A. V. Efremov, Phys. Rev. B 79, 054418 (2009); A. A. Mozhegorov, A. E. Nikiforov, A. V. Larin, A. V. Efremov, L. É. Gonchar', and P. A. Agzamova, Fiz. Tverd. Tela 50, 1724 (2008) [Phys. Solid State 50, 1795 (2008)].

${ }^{24}$ A. E. Nikiforov, S. E. Popov, and S. Yu. Shashkin, Phys. Met. Metallogr. 87, 97 (1999).

${ }^{25}$ L. E. Gontchar and A. E. Nikiforov, Phys. Rev. B 66, 014437 (2002); Zh. Eksp. Teor. Fiz. 123, 575 (2003) [J. Exp. Theor. Phys. 96, 510 (2003)].

${ }^{26}$ A. E. Nikiforov, S. Yu. Shashkin, M. L. Levitan, and T. H. Agamalyan, Phys. Status Solidi B 118, 419 (1988).

${ }^{27}$ A. E. Nikiforov, S. Yu. Shashkin, and A. I. Krotkii, Phys. Status Solidi B 98, 289 (1980).

${ }^{28}$ L. E. Gontchar and A. E. Nikiforov, J. Magn. Magn. Mater. 300, e167 (2006). 